\title{
Thermally assisted hydrolysis and alkylation of lignins in the presence of tetra-alkylammonium hydroxides
}

\author{
F. Martín *, J.C. del Río, F.J. González-Vila, T. Verdejo
}

Instituto de Recursos Naturales y Agrobiologia de Sevilla, C.S.I.C., P.O. Box 1052, 41080 Seville, Spain

Received 10 October 1994; accepted 2 February 1995

\section{Abstract}

Three different milled lignins isolated from bamboo, pine and beech, corresponding to the three different structural groups, have been subjected to pyrolysis in the presence of tetramethylammonium hydroxide (TMAH) and tetrabutylammonium hydroxide (TBAH). Pyrolysis of the lignins in the presence of TMAH releases the methyl esters and methyl ethers of the different lignin monomers. Several moieties bearing carboxylic acid groups, not previously detected using pyrolytic techniques, could be released from the three lignins, suggesting that the presence of these moieties in the lignin structure have been underestimated.

Keywords: Milled wood lignins; Pyrolysis; Pyrolysis-methylation; Thermally assisted hydrolysis and methylation; Tetrabutylammonium hydroxide; Tetramethylammonium hydroxide

\section{Introduction}

Pyrolysis-gas chromatography/mass spectrometry (Py-GC/MS) has been widely used in the structural characterization of lignin and lignin-containing materials [1-9]. The technique allows the identification of the different lignin units and has also been found suitable for characterizing lignins in the environment [10]. Pyrolysis of lignins produces a mixture of simple phenols resulting from the cleavages of 
ether and $\mathrm{C}-\mathrm{C}$ bonds, most of them retaining their substitution pattern [9]. However, conventional flash pyrolysis can underestimate the potential presence of units bearing carboxyl groups among the lignin structural 'building blocks', as occurs in humic substances [11,12]. In fact, flash pyrolysis of polymers containing benzenecarboxylic acid moieties leads to biased structural interpretation, as it has been proved that these moieties undergo decarboxylation under such conditions [11]. In the case of lignins, only a few carboxylic pyrolysis products are usually reported, although the presence of such groups have been confirmed by several spectroscopic and chemical wet methods [13]. In addition, the extent of the well-known role of cinnamic acids in the association between grass lignin and hemicellulose cannot be easily evidenced by analytical pyrolysis $[8,14]$.

In previous papers $[11,12,15]$ we have described the use of pyrolysis in the presence of tetramethylammonium hydroxide (TMAH) for the detection of benzenecarboxylic acids bearing units in the structure of humic substances, which otherwise undergo decarboxylation under conventional pyrolysis. This technique avoids decarboxylation, and produces methyl esters of carboxylic acids and methyl ethers of phenolic compounds. During the last few years this procedure has been wrongly termed simultaneous pyrolysis methylation (SPM) to distinguish it from pyrolytic methylation, which is frequently used to derivatize free fatty acids and triglycerides in the injection port of the gas chromatograph [16,17]. However, several authors have demonstrated that SPM consists of a thermally assisted chemolysis rather than a true pyrolysis followed by the methylation of the released pyrolysis products $[11,18,19]$. Therefore, the procedure has recently been more correctly termed thermally assisted hydrolysis and methylation (THM) [19], although the term pyrolysis-methylation seems to be more popular among the different authors. THM has been successfully applied to assess the structure of different natural and artificial polymers such as cutins, natural resinites, humic acids, alkyd resins, polyester fibres and rosin-based resins [11,12,15,18-28]. Pyrolysismethylation of bamboo milled wood lignin (MWL) has also been recently reported [29].

In similar terms, pyrolysis-methylation has been successfully applied to the monitoring of the structural changes undergone by lignin during the coalification process [15]. However, pyrolysis-methylation has a significant drawback in structural studies of degraded lignins which might have suffered from demethylation, such as occurs during coalification [10]. Since degraded lignins may contain both methoxyl and free hydroxyl groups which will become methylated upon pyrolysis-methylation, the latter cannot be distinguished from the original methoxyl groups present in the macromolecule. To avoid this problem, we introduce in this paper the technique of pyrolysis in the presence of tetrabutylammonium hydroxide (TBAH), which will produce butyl derivatives of the hydroxyl and carboxyl units, to differentiate between the free hydroxyl groups and the originally present methoxyl groups in the structure of degraded lignin.

The aim of this work is, therefore, to assess whether the procedure of thermally assisted hydrolysis and alkylation could provide a better understanding of the 
macromolecular structure of different lignins based upon pyrolysis techniques. Three different MWLs isolated from bamboo, pine and beech, and corresponding to the three different structural groups, were selected for pyrolysis in the presence of tetra-alkylammonium hydroxides. MWLs are considered to be, in many respects, almost identical to the original lignins, although they represent only $30-50 \%$ of the total lignin components, and generally contains small amounts of associated carbohydrates [13]. It should be taken into account that although MWLs do not fully represent natural lignins, this is the most useful isolated procedure for structural studies on lignin.

\section{Material and methods}

Three MWLs isolated from bamboo (Bamboo sp.), pine (Pinus pinea) and beech (Fagus silvatica) were selected for this study. These samples have been previously analyzed by conventional pyrolysis [1].

\subsection{Pyrolysis-gas chromatography-mass spectrometry}

For pyrolysis in the presence of TMAH and TBAH, the lignin samples were dispersed in the minimum amount of salts (25\% aqueous solution) and dried in a desiccator overnight, the latter being necessary to avoid the presence of water in the column. The syrups were placed on the ribbon foil of the CDS pyroprobe and heated to $500^{\circ} \mathrm{C}$ for $10 \mathrm{~s}$. Separation of the pyrolysis products was achieved by a fused silica column (DB-FFAP; J\&W Scientific) of $30 \mathrm{~m}$ length and $0.2 \mathrm{~mm}$ i.d. The gas chromatograph (Hewlett-Packard HP-5890) was programmed from 40 to $250^{\circ} \mathrm{C}$ at the rate of $6^{\circ} \mathrm{C} \mathrm{min}^{-1}$. Helium was used as carrier gas. The mass spectrometer (HP 5988 A) was set a $70 \mathrm{eV}$. Identification was achieved by mass fragmentography, library search and comparison with literature data. When possible, the identifications were accomplished by comparison with authentic standards.

\section{Results and discussion}

Conventional pyrolysis of the three MWL samples selected for this study did not reveal the presence of benzenecarboxylic acid moieties [1]. However, it is known that conventional pyrolytic conditions do not give evidence of the presence of aromatic acids as structural units owing to decarboxylation processes [11,27], as mentioned above, and thus their detection in the lignin structure might have been highly biased. In order to shed further light on the structural studies of lignin, the three MWLs selected for this study were subjected to pyrolysis derivatization with TMAH and TBAH. 


\subsection{Pyrolysis-methylation of lignins}

Fig. 1 shows the total ion chromatograms of the thermal degradative products obtained after the pyrolysis of the different lignins in the presence of TMAH. The main compounds identified, together with their relative abundances, are listed in Table 1.

The pyrograms of the three lignins are very different, and these differences seem to concern the relative amounts of the substituted cinnamyl alcohol precursors.

The bamboo lignins have a predominance of compounds derived from $p$-hydroxyphenyl units, in agreement with data from conventional pyrolysis. The main distinctive compound identified in the pyrogram of the bamboo lignin is the methyl derivative of $p$-hydroxycinamic acid (28). The pine lignin (representative of softwood) is composed exclusively of guaiacyl units. Thus, the major pyrolysis compounds released after pyrolysis-methylation corresponded to dimethoxybenzene derivatives. In the pyrogram of the beech lignin (representative of hardwood) there is not a remarkable predominance of compounds belonging to syringly units over those belonging to guaiacyl units, as expected, and both types of structural units were released.

It is interesting to point out the large amounts of compounds with the carboxyl groups, including two isomers of benzenedicarboxylic acids (appearing as methyl esters), released after the pyrolysis-methylation of the three lignins. These compounds were not previously detected by conventional pyrolysis owing to decarboxylation processes [11,27]. Different types of carboxylic units such as benzenecarboxylic acids, benzeneacetic acids and benzenepropenoic acids (cinnamic acids), were released. This indicated that the presence of carboxylic groups in the structure of lignins has previously been underestimated by conventional pyrolysis.

A series of compounds with base peak at $m / z 181$ or 211 in their mass spectra were detected in the pine and beech lignins respectively. It appears that these compounds are derived either from guaiacyl (base peak at $\mathrm{m} / \mathrm{z} 181$ ) or syringyl (base peak at $m / z 211$ ) units. Two isomers of two different compounds (Nos. 27/29 and 36/38) were tentatively identified as the trimethyl derivatives of guaiacyl-glycerol (1-(3,4-dimethoxyphenyl)glycerol trimethyl ether) and syringyl-glycerol (1-(3,4,5-trimethoxyphenyl)glycerol trimethyl ether), respectively. Guaiacyl-glycerol appears in both pine and beech lignin, whereas the syringyl-glycerol was released only from the beech lignin. These compounds are among the most abundant moieties released from the pine and beech lignin. As far as we known this is the first time that these units, although proposed in all lignin models, have been released as such by pyrolytic techniques. They have been reported, however, as hydrolytic products of lignins [30].

\subsection{Pyrolysis-butylation of lignins and standard compounds}

Table 2 presents data of the behaviour of the cinnamic acids and cinnamyl alcohols against conventional pyrolysis, pyrolysis-methylation and pyrolysis-butylation. Cinnamic acids undergo decarboxylation under conventional 

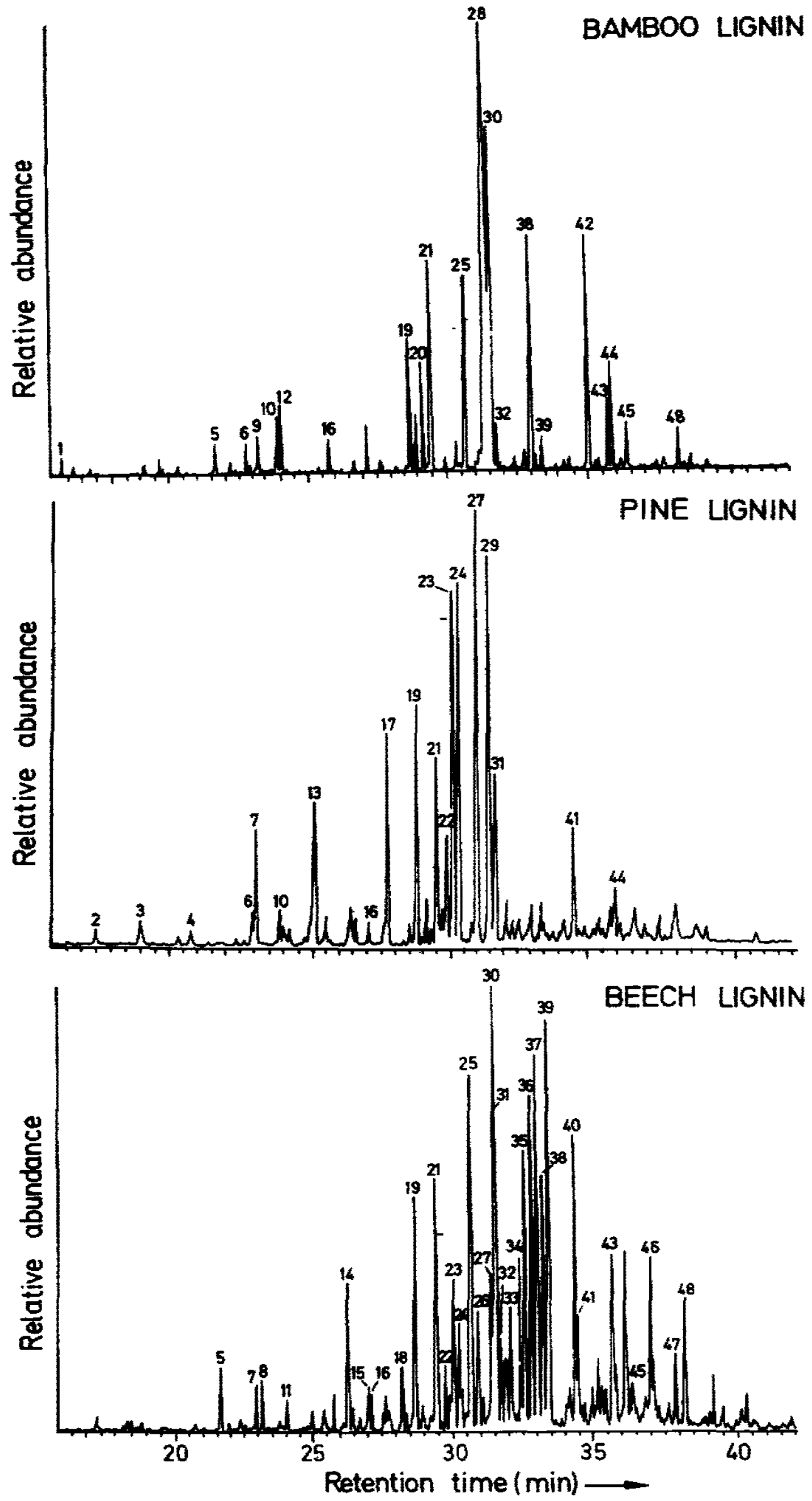

Fig. 1. Total ion chromatograms of the thermal degradation products obtained after pyrolysis of the bamboo, pine and beech lignins in the presence of TMAH. Numbers refer to the compounds listed in Table 1 . 
Table 1

Identification of the compounds released in the pyrolysis-methylation of lignins

\begin{tabular}{|c|c|c|c|c|}
\hline Number & Compound & Bamboo & Pine & Beech \\
\hline 1 & 1-Ethenyl-4-methoxybenzene & + & - & - \\
\hline 2 & 1,2-Dimethoxybenzene & - & + & - \\
\hline 3 & 3,4-Dimethoxytoluene & - & + & - \\
\hline 4 & 1-(3,4-Dimethoxyphenyl)ethanone & - & + & - \\
\hline 5 & 1,2,3-Trimethoxybenzene & + & - & + \\
\hline 6 & 4-Methoxybenzaldehyde & + & + & - \\
\hline 7 & 4-Ethenyl-1,2-dimethoxybenzene & - & ++ & + \\
\hline 8 & 1-Methyl-3,4,5-trimethoxybenzene & - & - & + \\
\hline 9 & Unknown & + & - & - \\
\hline 10 & 4-Methoxybenzoic acid methyl ester & + & + & - \\
\hline 11 & 1-(3,4,5-Trimethoxyphenyl)ethanone & - & - & + \\
\hline 12 & Unknown & + & - & - \\
\hline 13 & 3,4-Dimethoxybenzenemethanol methyl ether & - & ++ & - \\
\hline 14 & 1-Vinyl-3,4,5-trimethoxybenzene & - & - & ++ \\
\hline 15 & Benzenedicarboxylic acid dimethyl ester & - & - & + \\
\hline 16 & Isomer 15 & + & + & + \\
\hline 17 & 3,4-Dimethoxybenzeneacetic acid methyl ester & - & ++ & - \\
\hline 18 & 1-(3,4,5-Trimethoxy)benzenemethanol methyl ether & - & - & + \\
\hline 19 & 3,4-Dimethoxybenzaldehyde & ++ & ++ & ++ \\
\hline 20 & Isomer 28 & ++ & - & - \\
\hline 21 & 3,4-Dimethoxybenzoic acid methyl ester & ++ & ++ & ++ \\
\hline 22 & 1-Propenyl-3,4,5-trimethoxybenzene & - & + & + \\
\hline 23 & 1-(3,4-Dimethoxy phenyl)propanone & - & +++ & ++ \\
\hline 24 & Isomer 23 & - & +++ & + \\
\hline 25 & 3,4,5-Trimethoxybenzaldehyde & ++ & - & +++ \\
\hline 26 & Unknown & - & - & + \\
\hline 27 & 1-(3,4-Dimethoxyphenyl)glycerol trimethyl ether & - & +++ & ++ \\
\hline 28 & 3-(4-Methoxyphenyl)-2-propenoic acid methyl ester & +++ & - & - \\
\hline 29 & Isomer 27 & - & +++ & - \\
\hline 30 & 3,4,5-Trimethoxybenzoic acid methyl ester & $++t$ & - & +++ \\
\hline 31 & 3-(3,4-Dimethoxyphenyl)-2-propenol methyl ether & - & $+t$ & +++ \\
\hline 32 & 1-(3,4,5-Trimethoxyphenyl)ethanone & + & - & + \\
\hline 33 & 3-(3,4,5-Trimethoxyphenyl)-2-propenol methyl ether & - & - & + \\
\hline 34 & 1-(3,4,5-Trimethoxyphenyl)propionaldehyde & - & - & + \\
\hline 35 & Isomer 33 & - & - & ++ \\
\hline 36 & 1-(3,4,5-Trimethoxyphenyl)glycerol trimethyl ether & - & - & $++t$ \\
\hline 37 & 1-(3,4,5-Trimethoxyphenyl)propanone & - & - & +++ \\
\hline 38 & Isomer 36 & ++ & - & ++ \\
\hline 39 & 3-(3,4-Dimethoxyphenyl)-2-propenoic acid methyl ester & + & - & +++ \\
\hline 40 & Isomer 33 & - & - & ++ \\
\hline 41 & Unknown & - & ++ & + \\
\hline 42 & 2,3,4,5-Tetramethoxyphenylacetic acid methyl ester & ++ & - & - \\
\hline 43 & 3-(3,4-Dimethoxyphenyl)propanoic acid methyl ester & + & - & ++ \\
\hline 44 & Isomer 39 & ++ & + & - \\
\hline 45 & 3-(3,4,5-Trimethoxyphenyl)propanoic acid methyl ester & + & - & + \\
\hline 46 & 4,4'-Dimethoxydimethylstilbene & - & - & ++ \\
\hline 47 & 3-(3,4,5-Trimethoxy)benzenepropanol methyl ether & - & - & + \\
\hline 48 & 3-(3,4,5-Trimethoxyphenyl)-2-propenoic acid methyl ester & + & - & ++ \\
\hline
\end{tabular}

(-): not detected

$(+),(++),(+++)$ : low, medium and high amounts, respectively 
Table 2

Pyrolytic behaviour of some standard lignin monomeric units

\begin{tabular}{|c|c|c|c|}
\hline Compound & Conventional pyrolysis & Pyrolysis-methylation & Pyrolysis-butylation \\
\hline $\begin{array}{l}\text { 3-(4-Hydroxyphenyl)-2-propenoic acid } \\
\text { (p-coumaric acid) }\end{array}$ & 4-Vinylphenol & $\begin{array}{l}\text { 3-(4-Methoxyphenyl)-2. } \\
\text { propenoic acid methyl } \\
\text { ester }\end{array}$ & $\begin{array}{l}\text { 3-(4-Butoxyphenyl)-2-propenoic } \\
\text { acid butyl ester }+4 \text {-vinyl- } \\
\text { butoxybenzene }(1: 1)\end{array}$ \\
\hline $\begin{array}{l}\text { 3-(3-Methoxy-4-hydroxyphenyl)-2- } \\
\text { propenoic acid } \\
\text { (ferulic acid) }\end{array}$ & 4-Vinyl-2-methoxyphenol & $\begin{array}{l}\text { 3-(3,4-Dimethoxyphenyl)- } \\
\text { 2-propenoic acid methyl ester }\end{array}$ & $\begin{array}{l}\text { 3-(3-Methoxy-4-butoxyphenyl)- } \\
\text { 2-propenoic acid butyl ester } \\
\text { + 4-vinyl-2-methoxybutoxy- } \\
\text { benzene }(1: 5)\end{array}$ \\
\hline $\begin{array}{l}\text { 3-(3,5-Dimethoxy-4-hydroxyphenyl)- } \\
\text { 2-propenoic acid } \\
\text { (sinapinic acid) }\end{array}$ & $\begin{array}{l}\text { 4-Vinyl-2,6-dimethoxy- } \\
\text { phenol }\end{array}$ & $\begin{array}{l}\text { 3-(3,4,5-Trimethoxyphenyl)- } \\
\text { 2-propenoic acid methyl ester }\end{array}$ & $\begin{array}{l}\text { 3-(3,5-Dimethoxy-4-butoxyphenyl)-2- } \\
\text { propenoic acid butyl ester } \\
+4 \text {-vinyl-2,6-dimethoxybutoxy- } \\
\text { benzene }(1: 10)\end{array}$ \\
\hline $\begin{array}{l}\text { 3-(4-Hydroxyphenyl)-2-propenol } \\
\text { (p-coumaryl alcohol) }\end{array}$ & $\begin{array}{l}\text { 3-(4-Hydroxyphenyl)-2- } \\
\text { propenol }\end{array}$ & $\begin{array}{l}\text { 3-(4-Methoxyphenyl)-2. } \\
\text { propenol methyl ester }\end{array}$ & 3-(4-Butoxyphenyl)-2-propenol \\
\hline $\begin{array}{l}\text { 3-(3-Methoxy-4-hydroxyphenyl)- } \\
\text { 2-propenol } \\
\text { (coniferyl alcohol) }\end{array}$ & $\begin{array}{l}\text { 3-(3-Methoxy-4-hydroxy- } \\
\text { phenyl)-2-propenol }\end{array}$ & $\begin{array}{l}\text { 3-(3,4-Dimethoxyphenyl)-2- } \\
\text { propenol methyl ester }\end{array}$ & $\begin{array}{l}\text { 3-(3-Methoxy-4-butoxyphenyl)- } \\
\text { 2-propenol }\end{array}$ \\
\hline $\begin{array}{l}\text { 3-(3,5-Dimethoxy-4-hydroxy- } \\
\text { phenyl)-2-propenol } \\
\text { (sinapyl alcohol) }\end{array}$ & $\begin{array}{l}\text { 3-(3,5-Dimethoxy-4- } \\
\text { hydroxyphenyl)-2- } \\
\text { propenol }\end{array}$ & $\begin{array}{l}\text { 3-(3,4,5-Trimethoxyphenyl)- } \\
\text { 2-propenol methyl ester }\end{array}$ & $\begin{array}{l}\text { 3-(3,5-Dimethoxy-4-butoxyphenyl)- } \\
\text { 2-propenol }\end{array}$ \\
\hline
\end{tabular}



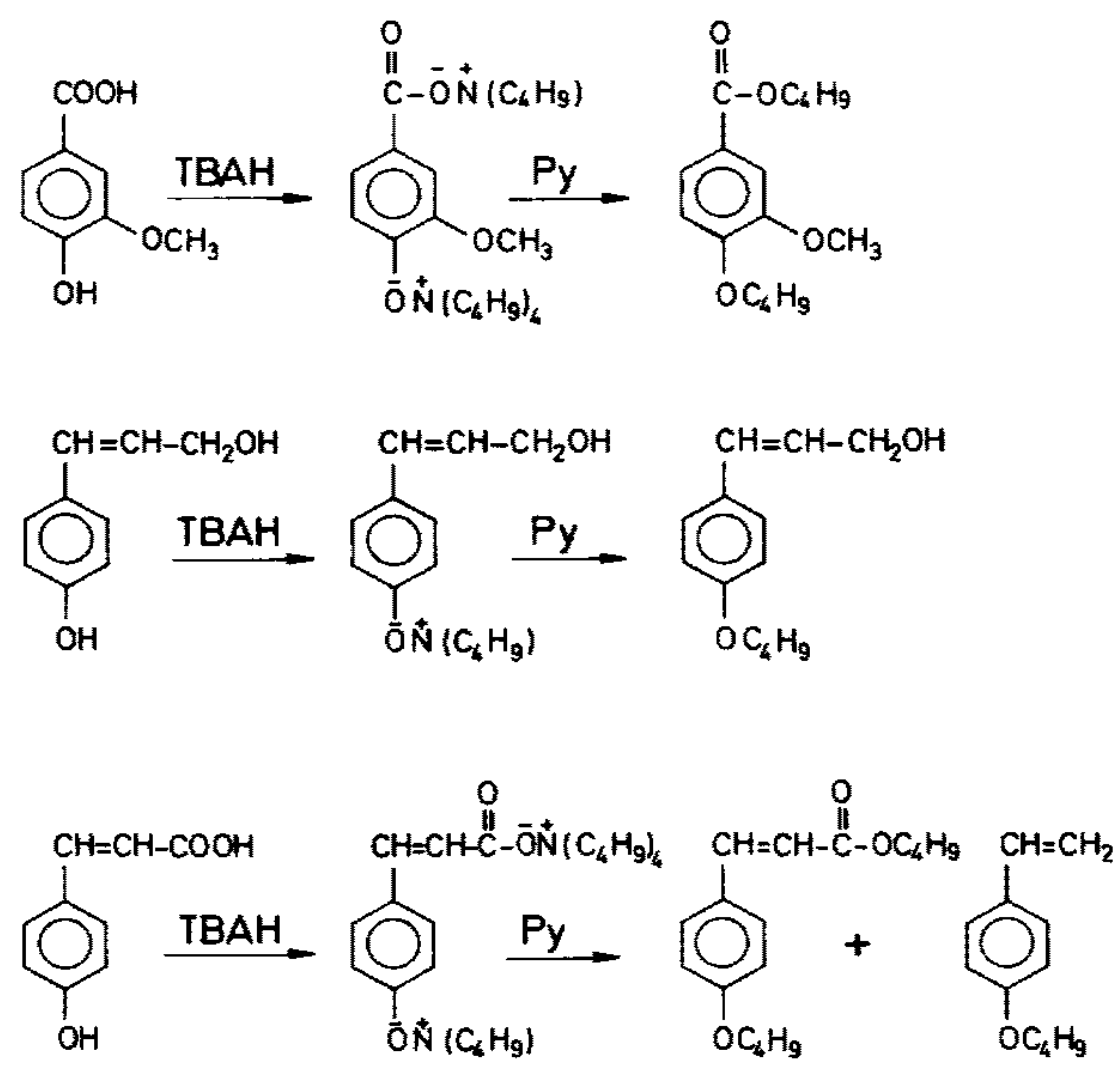

Fig. 2. Suggested mechanism for the pyrolytic butylation of different lignin units.

pyrolysis, and yield the corresponding vinylphenols. However, pyrolysismethylation and pyrolysis - butylation release the corresponding cinnamic acids as the methyl and butyl derivatives, respectively. Pyrolysis-butylation also releases the products arising from the decarboxylation, these becoming more predominant with increasing molecular weight of the cinnamic acids. The cinnamyl alcohols are released as such on conventional pyrolysis and with the free aliphatic and aromatic $\mathrm{OH}$ fully methylated ethers on pyrolysis-methylation. In the pyrolysis-butylation, only the phenolic $\mathrm{OH}$ groups become butylated, while the aliphatic alcohols remain unsubstituted, probably owing to their low acidic character. Attempts to butylate the alcohol groups failed at three different pyrolysis temperatures (300,500 and $700^{\circ} \mathrm{C}$ ). According to these data, we suggest the mechanism shown in Fig. 2 for pyrolysis-butylation, similar to that reported for pyrolysis-methylation [11].

With respect to the mass spectra of the butyl derivatives, the fragmentation of the butyl ester involves loss of the butyl radical and transfer of one atom of hydrogen, giving the molecular ion of the original compounds [31]. The same mechanism seems to occur in the case of butyl-aryl ether, as reflected in Fig. 3. Therefore, the resulting mass spectrum of a butyl derivative will be characterized by the molecular ion, different fragments corresponding to loss of different butyl radicals and, finally, the characteristic fragments of the original non-butylated molecule. Fig. 4 shows the mass spectra of the butyl derivatives of the three cinnamic acids, ( $p$-coumaric, ferulic and sinapinic acids).

The total ion chromatograms of the pyrolysis products released from the different lignins in the presence of TABH are shown in Fig. 5. The identities of the compounds, released as butyl ether and butyl ester derivatives, are listed in Table 
<smiles>CCCCCCCCCCC(C)CCOC(=O)c1ccccc1</smiles>

Fig. 3. Fragmentation pattern of the butyl derivatives in their mass spectra.

3. As in the case of pyrolysis-methylation, there are evident differences among the different pyrograms after pyrolysis-butylation.

Bamboo lignin released major peaks derived from the $p$-hydroxyphenyl structure, typical of this type of lignin, and corresponding to the butyl derivatives of
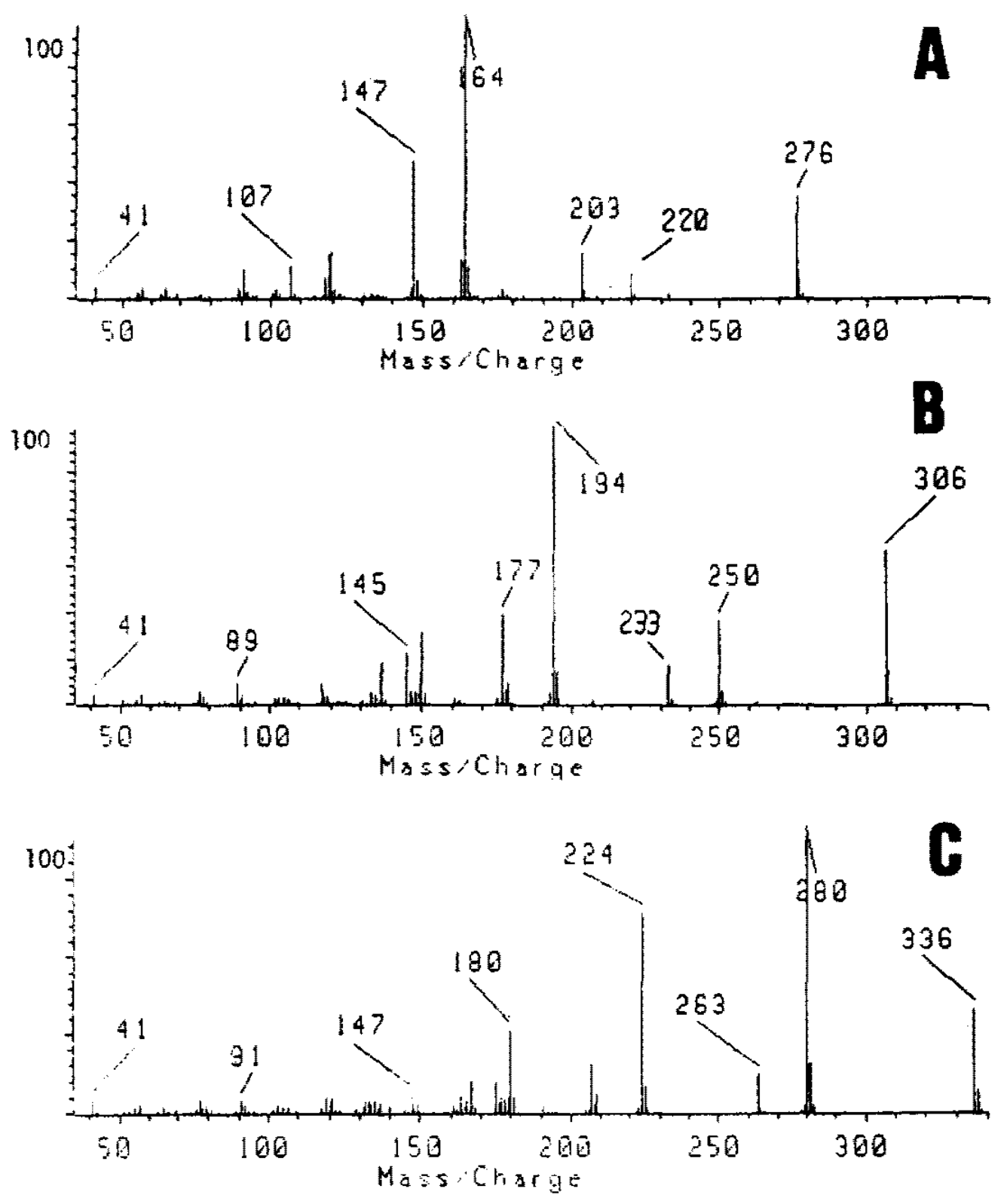

Fig. 4. Mass spectra of: (A) 3-(4-hydroxyphenyl)-2-propenoic acid ( $p$-coumaric acid), (B) 3-(3-methoxy4-hydroxyphenyl)-2-propenoic acid (ferulic acid) and (C) 3-(3,5-dimethoxy-4-hydroxyphenyl)-2propenoic acid (sinapinic acid) as butyl esters and butyl ethers). 

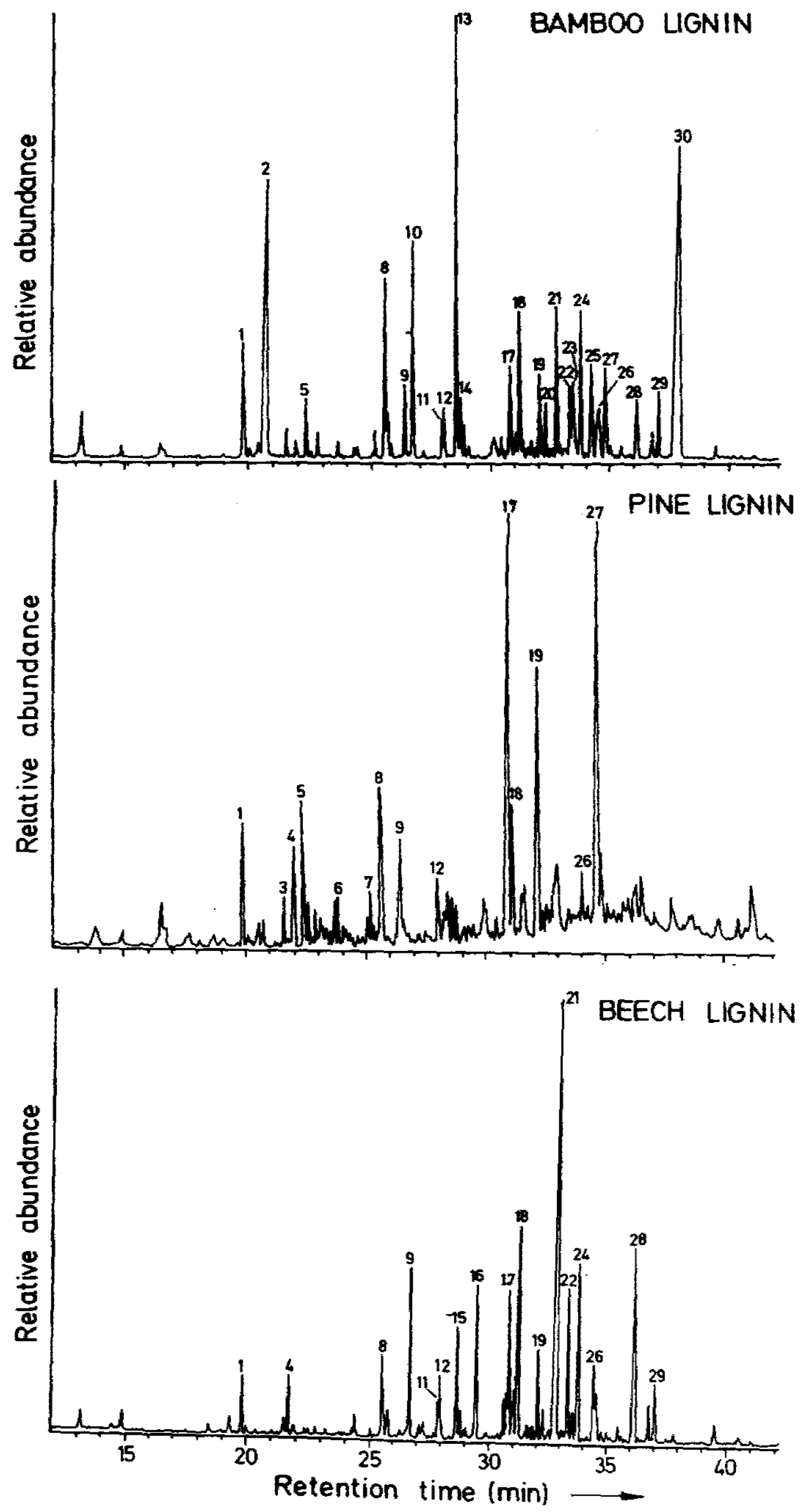
Fig. 5 . Total ion chromatograms of the products released in the pyrolysis-butylation of different lignins.
Numbers refer to the compounds in Table 3 . 
vinylphenol (2), 4-methylbenzaldehyde (13) and $p$-coumaric acid (30). For the pine lignin, only guaicyl compounds were identified. The major compounds released corresponded to the butyl derivatives of 4-hydroxy-3-methoxybenzaldehyde (vanillin) (17), 1-(4-hydroxy-3-methoxyphenyl) ethanone (19) and dihydroconiferyl alcohol (27), the latter being the only alcohol detected in this lignin. Coniferyl alcohol was released in minor amounts. In the case of the beech lignin, the compounds released presented both guaiacyl and syringyl structures with a ratio close to unity. The major monomer released (as butyl ether) was 4-hydroxy3,5-dimethoxyphenylbenzaldehyde (21). Sinapyl alcohol was detected with butylation of only the phenolic $\mathrm{OH}$.

Table 3

Identification of the compounds released in the pyrolysis-butylation of lignins

\begin{tabular}{|c|c|c|c|c|}
\hline Number & Compound & Bamboo & Pine & Beech \\
\hline 1 & 2-Methoxyphenol & $+t$ & ++ & + \\
\hline 2 & 4-Vinylphenol & $++t$ & - & - \\
\hline 3 & 1,4-Dimethoxybenzene & - & + & - \\
\hline 4 & Isomer of 3 & - & ++ & + \\
\hline 5 & Phenol & + & ++ & - \\
\hline 6 & 4-Methylphenol & - & + & - \\
\hline 7 & 4-(1-Propenyl)phenol & - & + & - \\
\hline 8 & 2-Methoxy-4-vinylphenol & ++ & ++ & + \\
\hline 9 & 4-Hydroxybenzaldehyde & - & ++ & $+t$ \\
\hline 10 & 2,4-Dimethoxyphenol & ++ & - & - \\
\hline 11 & 2-Methoxy-4-(1-propenyl)phenol & + & - & + \\
\hline 12 & 1-(4-Hydroxy-3-methoxyphenyl)ethanol ${ }^{a}$ & + & + & + \\
\hline 13 & 4-Methylbenzaldehyde & +++ & - & - \\
\hline 14 & 2,6-Dimethoxyphenol & + & - & - \\
\hline 15 & 2-Methoxy-4-methylphenol & - & - & ++ \\
\hline 16 & 3,4-Dimethoxybenzoic acid methyl ester & - & - & ++ \\
\hline 17 & 4-Hydroxy-3-methoxybenzaldehyde & + & +++ & ++ \\
\hline 18 & 4-Hydroxy-3-methoxybenzoic acid methyl ester & ++ & ++ & ++ \\
\hline 19 & 1-(4-Hydroxy-3-methoxyphenyl)ethanone & + & ++ & + \\
\hline 20 & Unknown & + & - & - \\
\hline 21 & 4-Hydroxy-3,5-dimethoxybenzaldehyde & ++ & - & +++ \\
\hline 22 & 2,6-Dimethoxy-4-(2-propenyl)phenol & + & - & ++ \\
\hline 23 & 1-(3,4-Trimethoxyphenyl)ethanone & + & - & - \\
\hline 24 & 1-(4-Hydroxy-3,5-dimethoxyphenyl)ethanone & ++ & - & ++ \\
\hline 25 & 3-(4-Hydroxyphenyl)-2-propenoic acid methyl ester & + & - & - \\
\hline 26 & 4-Hydroxy-3-methoxybenzoic acid & + & + & + \\
\hline 27 & 3-(4-Hydroxy-3-methoxyphenyl)propanol (a) & + & +++ & - \\
\hline 28 & 4-Hydroxy-3,5-dimethoxybenzoic acid & + & - & ++ \\
\hline 29 & 3-(4-Hydroxy-3,5-dimethoxyphenyl)propanone & + & - & + \\
\hline 30 & 3-(4-Hydroxyphenyl)-2-propenoic acid & $+t+$ & - & - \\
\hline
\end{tabular}

Compounds are referred as $n$-butyl esters and $n$-butyl ethers (except for $3,4,13$ ).

$(-)$ : not detected. $(+,++,+++)$ : low, medium, high relative abundances, respectively.

${ }^{a}$ The aliphatic alcohol is free and not butylated. 
As in the case of pyrolysis-methylation, the most striking feature observed after pyrolysis-butylation was the identification of carboxyl-bearing units among the compounds released in the pyrolysis of the three lignin samples $(16,26,28,30)$. These carboxylic groups were released in higher amounts from the beech lignin. Two different types of carboxylic groups were identified: benzenecarboxylic acids $(16,26,28)$ and cinnamic acids $(30)$. Butyl derivatives of cinnamic acids, such as $p$-coumaric acid (30), were only found in bamboo lignin. However, several carboxylic groups were released as methyl esters on pyrolysis-butylation $(18,25)$. The carboxyl-bearing units may be free or esterified in the original macromolecule. This could explain the release of some carboxylic moieties as methyl esters $(16,18,25)$ which would be as such in the lignin structure. In fact, the 3,4-dimethoxybenzoic acid methyl ester (peak 21 in Table 1) released after pyrolysis-methylation of the pine lignin, after pyrolysis-butylation is released with butylation of the hydroxyl group in position C-4 butylated and methylation of the carboxyl group (peak 18 in Table 3). This example shows that this carboxyl group was originally methylated in the lignin structure.

\section{Conclusions}

Pyrolysis in the presence of TMAH and TBAH provides valuable complementary information on the structure of different classes of lignins. Both are particularly effective for the detection of carboxyl-bearing compounds. In addition, pyrolysis-butylation seems to be appropriate for monitoring the changes undergone by the lignin during degradation processes involving demethylation by differentiating between original methoxyl groups and free phenolic groups.

\section{Acknowledgement}

The authors wish to thank the CICyT (Spanish Direccion General de Investigación Científica y Técnica; Project PB91-0074) for financial support.

\section{References}

[1] F. Martín, C. Sáiz-Jiménez and F.J. González-Vila, Holzforschung, 33 (1979) 210.

[2] T.J. Fullerton and R.A. Franich, Holzforschung, 37 (1983) 267.

[3] C. Saiz-Jimenez and J.W. de Leeuw, Org. Geochem., 10 (1986) 869.

[4] J.J. Boon, A.D. Pouwels and G.B. Eijkel, Biochem. Soc. Trans., 15 (1987) 170.

[5] A.D. Pouwels and J.J. Boon, J. Wood Chem. Technol., 7 (1987) 197.

[6] S. Salo, S. Niemela, S. Elomaa and M. Lindberg, Holzforschung, 43 (1989) 257.

[7] O. Faix, D. Meier and I. Fortman, Holzst. Rohst. Werkst., 48 (1990) 281.

[8] J. Ralph and R.D. Hatfield, J. Agric. Food Chem., 39 (1991) 1426.

[9] E.R.E. van de Hage, M.M. Mulder and J.J. Boon, J. Anal. Appl. Pyrolysis, 25 (1993) 149.

[10] P.G. Hatcher, Org. Geochem., 16 (1990) 959. 
[11] F. Martín, F.J. González-Vila, J.C. del Río and T. Verdejo, J. Anal. Appl. Pyrolysis, 28 (1994) 71. [12] F. Martin, J.C. del Río, F.J. González-Vila and T. Verdejo, J. Anal. Appl. Pyrolysis, 31 (1995) 75.

[13] W.G. Glasser and S. Sarkanen (Eds.), Lignin. Properties and Materials, ACS Symp. Ser. 397, American Chemical Society, Washington, DC, 1989, p. 545.

[14] M.C. Terrón, M.L. Fidalgo, A.E. González, G. Almendros and C. Galleti, J. Anal. Pyrolysis, 27 (1993) 57.

[15] J.C. de Rio, F.J. Gonzalez-Vila, F. Martin and T. Verdejo, Org. Geochem., 22 (1994) 885.

[16] W.C. Kossa, J. MacGee, S. Ramachandran and A.J. Webber, J. Chromatogr. Sci., 17 (1979) 177.

[17] L.D. Metcalf and C.N Wang, J. Chromatogr. Sci., 19 (1981) 530.

[18] J.W. de Leeuw and M. Baas, J. Anal. Appl. Pyrolysis, 26 (1993) 175.

[19] J.M. Challinor, J. Anal. Appl. Pyrolysis, 29 (1994) 223.

[20] J.M. Challinor, J. Anal. Appl. Pyrolysis, 16 (1989) 323.

[21] J.M. Challinor, J. Anal. Appl. Pyrolysis, 18 (1991) 233.

[22] J.M. Challinor, J. Anal. Appl. Pyrolysis, 20 (1991) 15.

[23] J.M. Challinor, J. Anal. Appl. Pyrolysis, 25 (1993) 349.

[24] K. Anderson and R. Winans, Anal. Chem., 63 (1991) 2901.

[25] C. Saiz-Jimenez, B. Hermosin and J.J. Ortega-Calvo, Water Res., 27 (1993) 1693.

[26] P.G. Hatcher and D.J. Clifford, Org. Geochem., 21 (1994) 1081.

[27] C. Saiz-Jimenez, Environ. Sci. Technol., 28 (1994) 197.

[28] G. Chiavari, G. Torsi, D. Fabbri and G.C. Galletti, Analyst, 119 (1994) 1141.

[29] M.M. Mulder, E.R.E. van der Hage and J.J. Boon, Phytochem. Anal., 3 (1992) 165.

[30] K. Freudenberg and A.C. Neish (Eds.), Constitution and Biosynthesis of Lignin, Vol. 2, Molecular Biology, Biochemistry and Biophysics, Springer-Verlag, New York, 1968, p. 129.

[31] J.H. Beynon, R.A. Saunders and A.E. Williams (Eds.), The Mass Spectra of Organic Molecules, Elsevier, Amsterdam, 1968, p. 510. 